\title{
Permeabilidad del hormigón a los gases y líquidos. Nuevo método de determinación
}

\author{
S. ALEGRE, Ingeniero Industrial y J. A. LECHUGA, Ingeniero Quimico
}

CABI, S. A. (Barcelona)

\section{$R E S U M E N$}

Se presenta un nuevo método de medición de la impermeabilidad del hormigón a gases y liquidos, de ejecución muy sencilla. Se desarrolla su fundamento teórico y se correlacionan algunos resultados obtenidos con la resistencia a compresión del hormigón y con los componentes empleados en su dosificación.

De todo ello, se deduce la conveniencia de replantear los criterios actualmente empleados para cuantificar las prestaciones del hormigón.

\author{
$S U M M A R Y$
}

A new measurement system is presented for impermeability of concrete to liquids and gas, of very easy esecution. It's technical basis is developed, and some of the results obtained are related with the components used in it's dosification.

Therefore, the judgments actually used for the quantification of concrete's utilities must be restated.

\section{N T R O D U C C I O N}

Podria entenderse por permeabilidad, de forma genérica, el grado de dificultad que ofrece el hormigón a que los fluidos penetren en su interior. Esta dificultad está ligada a su compacidad, entendiendo por tal la fracción de volúmen hueco del hormigón. A su vez, estos huecos pueden ser accesibles (si están comunicados entre sí y con el exterior), o inaccesibles (sino lo están). Son los huecos accesibles los que condicionan la penetración de fluidos en el hormigón. Los conductos capilares que los unen son los caminos para esta penetración. El diámetro de estos capilares condiciona el fenómeno de la capilaridad del hormigón (como hormigón se entiende áridos y pasta). Los poros del árido correcto no deben influir en la permeabilidad del hormigón. Por todo ello, cabe plantearse lo siguiente: como la compacidad del hormigón puede determinarse fácilmente (por pesos específicos real y aparente), y si asimilamos poros inaccesibles a aire ocluido (que también se mide con facilidad), el disponer de un método de medición de la permeabilidad (que también sea sencillo), puede ampliar sensiblemente el conocimiento de la estructura interna del hormigón, lo que debe permitir prever con mayor conocimiento de causa su comportamiento en distintas circunstancias.

Una de estas circustancias sería la resistencia que oponga el hormigón a la penetración de fluidos agresivos externos, como por ejemplo el agua de mar. La permeabilidad, pues, condicionaria su durabilidad frente a ellos. Lo mismo sucedería con los agresivos que se encuentren ya en el interior del hormigón, pero que para desarrollar sus efectos precisen de la concurrencia de algún elemento ajeno al hormigón, y que por tanto deba penetrar en él, como por ejemplo el oxigeno para los sulfuros.

Otra circunstancia podría ser la penetración de elementos que, sin ser agresivos, pudieran llegar a serlo si cambian las condiciones de conservación. Por ejemplo el agua, en caso de que se congele.

De otra parte, suele admitirse que las propiedades del hormigón (en especial su resistencia) dependen esencialmente de su relación agua-cemento y de su compacidad. De los extensos 
estudios realizados (de los que después se darán algunos ejemplos) se induce la buena correiacion existente entre Resistencia y Permeabilidad (concepto ligado al de compacidad), pero se evidencia también la incoherente dependencia entre la Resistencia y la Relación Agua-Cemento, en los hormigones con adiciones (tanto si ésta se incorpora al cemento como al propio hormigon). Ello da pic a reflexionar sobre la vigencia de ios criterios actualmente empleados para cuantificar las prestaciones del hormigón, y a tenor de los datos experimentales deducir que, algunos de ellos, podrian considerarse obsoletos para hormigones que incorporan adiciones en su dosificación.

De cualquier forma la justificación del empleo de relaciones, entre varios componentes del hormigón para prever algunas prestaciones, parece ser precisamente la de no poderlas medir directamente. Pues bien, si dichas prestaciones pudieran medirse (o bien alguna variable ineludiblemente ligada a ellas), tales relaciones quedarian situadas en inferioridad con respecto a dicha nueva medición. Tal puede ser el caso de la Permeabilidad, con respecto a las circunstancias ya apuntadas.

\section{FUNDAMENTO DEL METODO PRESENTADO}

Se ha puesto a punto un método novedoso con importantes ventajas de tipo práctico. Se emplean los moldes cilindricos de $15 \times 30 \mathrm{~cm}$ habituales en el control de resistencia del hormigón, con lo que la muestra es suficientemente representativa; el instrumental empleado en la cjecución es muy sencillo, y casi todo lo suelen ya poseer los laboratorios de control; las probetas no necesitan de ningún tratamiento superficial; las condiciones de los ensayos son compatibles con la jornada laboral.

En la figura 1 se indica el tipo de probeta empleada para los ensayos de resistencia y permeabilidad que, al proceder del mismo hormigón, permiten correlacionar ambos resultados, realizados a una edad y tras una conservación iguales.

PROBETAS PARA CORRELACIONAR

PERMEABILIDAD Y RESISTENCIA A COMPRESION

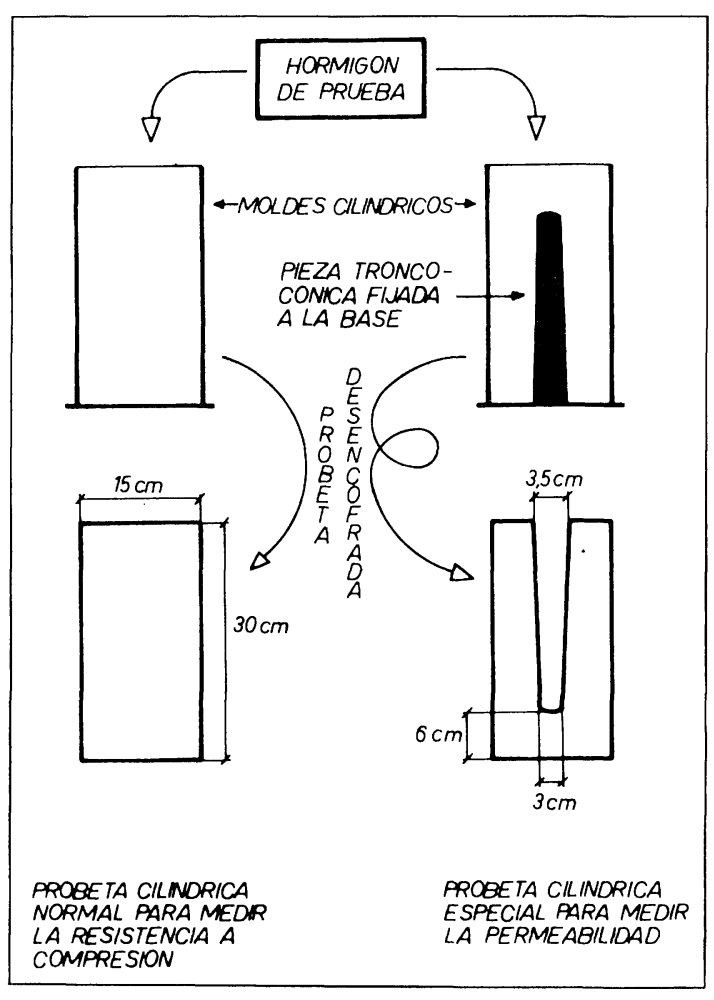

Fig. 1
SIMBOLOGIA EMPLEADA EN LA DESCRIPCION DEL METODO "DEL CALE"

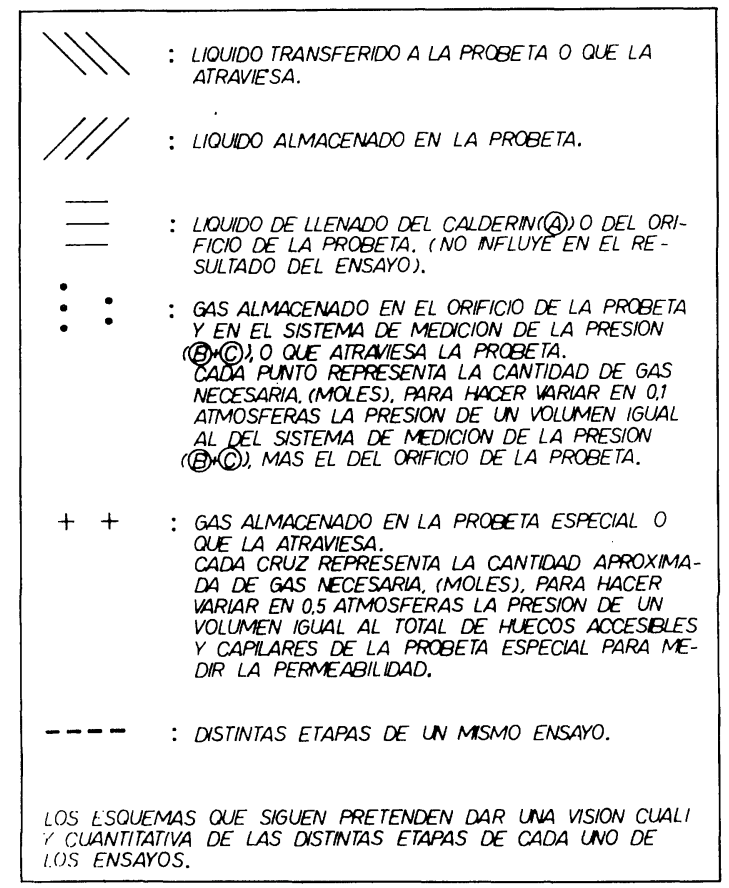

Fig. 2 
El utillaje empleado en la medición de la permeabilidad se indica en la figura 3. En los ensayos con liquidos se emplean las tres partes ( $(A)$, (B) y (C) ), mientras que en los ensayos con gases sólo dos ( (B) y (C) ), tal como indican las siguientes figuras. (La parte (A) puede desmontarse en cualquier momento para pesarla).

El método se basa en hacer circular un fluido desde el orificio de la probeta al exterior, impulsado por una sobrepresión en dicho orificio. En el caso de líquidos lo que se mide es la cantidad que atraviesa la probeta en un tiempo dado, manteniendo constante la presión en dicho orificio. En el caso de gases, lo que se hace es dar una cierta presión en el orificio, y medir el tiempo que tarda en descender dicha presión hasta otra, fijada en las condiciones experimentales. Un tercer ensayo que puede realizarse (absorción de liquidos) se lleva a cabo midiendo la cantidad de agua que penetra en la probeta ( trabajo.

Como la humedad de las probetas influye en todos los ensayos comentados, se han tomado dos situaciones extremas y fáciles de lograr: la saturación total (a presión de trabajo), y la sequedad total (tras un presecado de al menos un día a $70^{\circ} \mathrm{C}$ y un secado total a $115^{\circ} \mathrm{C}$ ).

En la figura 3 se indican los símbolos adoptados en las siguientes, para dar una idea gráfica de todo lo indicado.

En la figura 4 se indican las distintas etapas a seguir en la medición de la permeabilidad a los liquidos, cuyo resultado depende del tipo de hormigón ensayado.

En la figura 5 se esquematiza el ensayo de permeabilidad a los gases, dando una idea gráfica (que intenta ser cuantitativa) del trasiego de gas, durante el ensayo, distinguiendo la procedencia de cada cantidad parcial del gas. Se da una justificación (sobre la que después se profundizará) de que cada cantidad parcial de gas trasegado es constante, por lo que el tiempo empleado en ello depende del tipo de hormigón ensayado.

\section{UTILLAJE PARA LA MEDION DE LA PEMEABILIDAD}

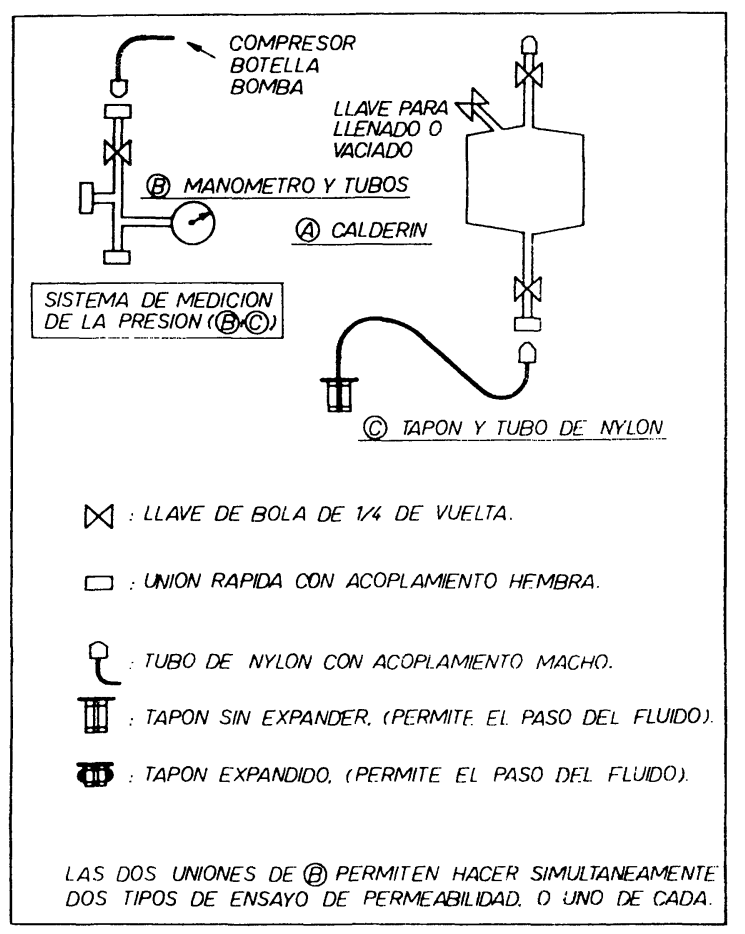

Fig. 3

\section{PERMEABILIDAD A LOS LIQUIDOS}

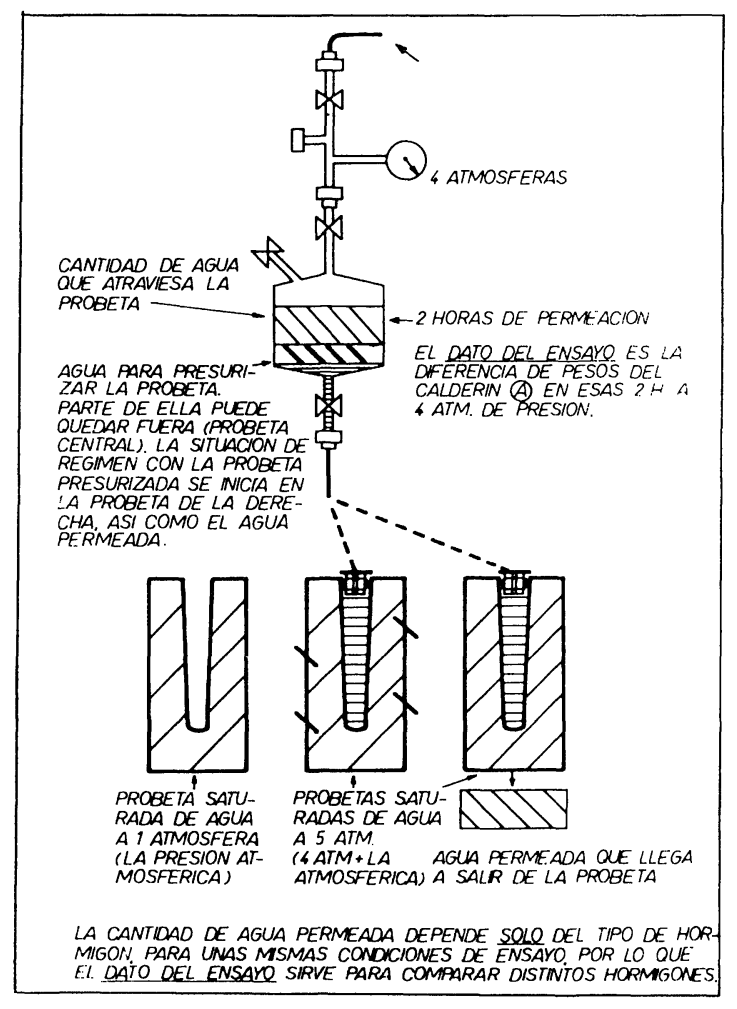

Fig. 4 
PERMEABILIDAD A LOS GASES

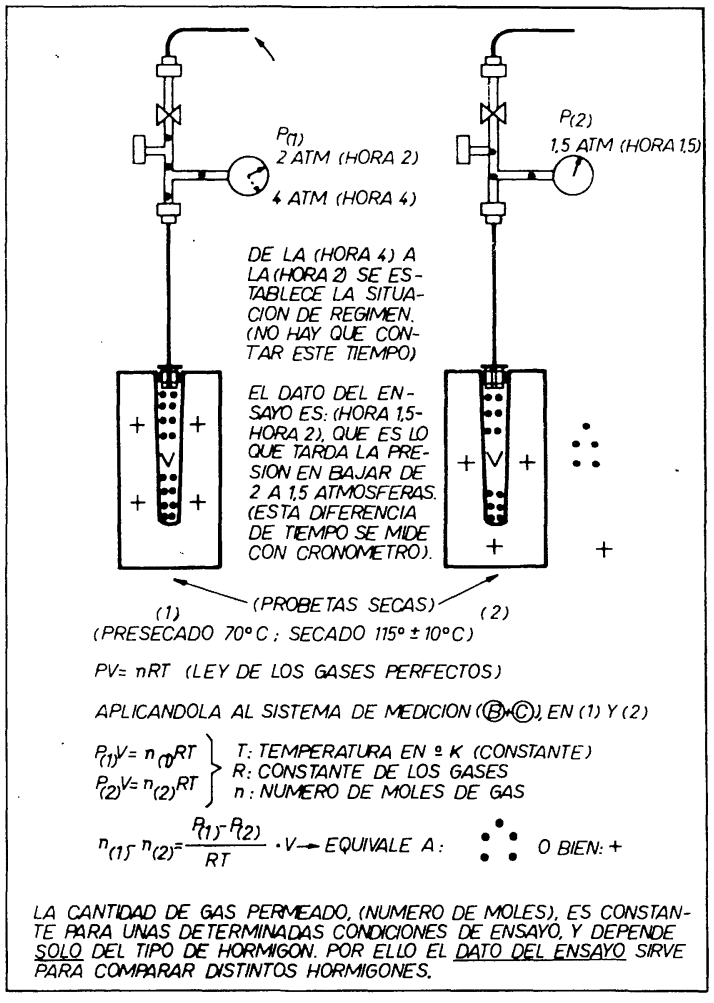

Fig. 5

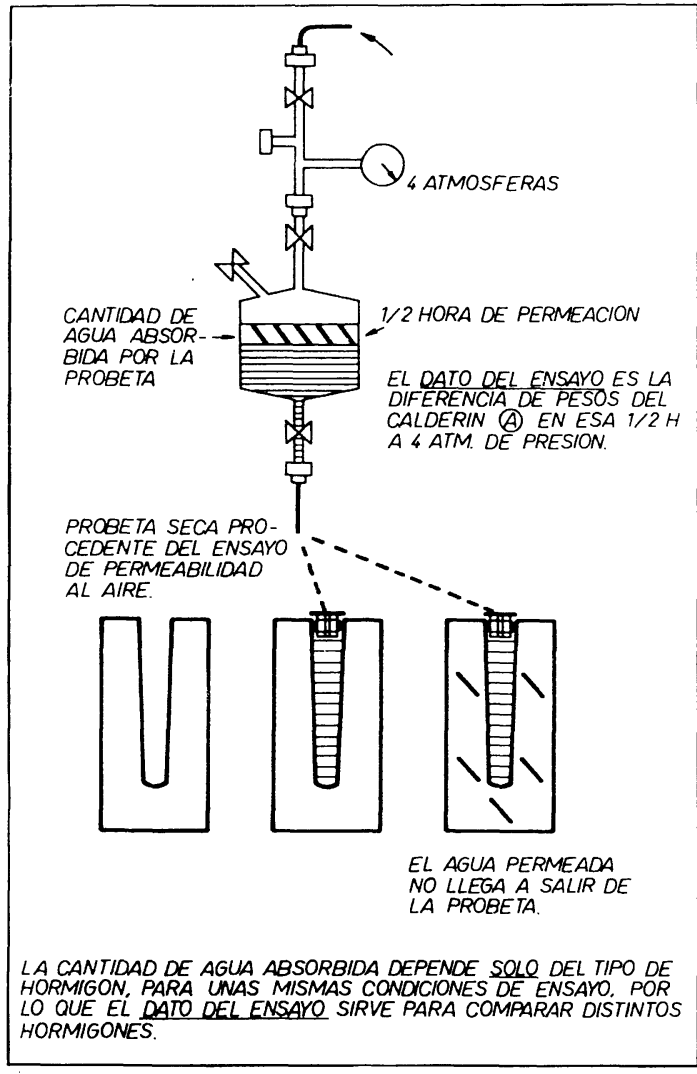

Fig. 6

En la figura 6 se indica la forma de realizar el llamado ensayo de absorción de líquidos, en el que la cantidad trasegada a la probeta, en un tiempo dado, debe ser menor que la máxima que ésta pueda absorber. Como el tiempo de permeación es constante, dicha cantidad depende del tipo de hormigón ensayado.

Es interesante recalcar que en este ensayo no se miden caudales, sino cantidades en un tiempo dado, o viceversa. Ello simplifica muchísimo el diseño del permeabilímetro.

Las condiciones experimentales, ya indicadas en las figuras anteriores, son las siguientes:

\begin{tabular}{|c|c|c|c|c|c|c|}
\hline & \multirow{2}{*}{$\begin{array}{l}\text { Presión de } \\
\text { trabajo }(*)\end{array}$} & \multicolumn{2}{|c|}{ Presión ensayo } & \multirow[b]{2}{*}{ Tiempo } & \multirow{2}{*}{$\begin{array}{c}\text { Liquido } \\
\text { permeado }\end{array}$} & \multirow{2}{*}{$\begin{array}{c}\text { Secado } \\
70^{\circ} \mathrm{C} 115^{\circ} \mathrm{C}\end{array}$} \\
\hline & & Inicial & Final & & & \\
\hline Perm. liq. & 4 Atm. & - & - & 2 horas & (DATO) & (Saturada a 4 Atm.) \\
\hline Perm. gas. & 4 Atm. & 2 Atm. & 1,5 Atm. & (DATO) & - & SI \\
\hline Absor. liq. & 4 Atm. & - & - & $1 / 2$ hora & (DATO) & SI \\
\hline
\end{tabular}

(*) Sobre la atmosférica.

Como al referenciar los datos habria que mencionar todas las condiciones experimentales, se ha preferido dar un nombre al método, lo que ya implica dichas condiciones. Se denominó método "del cale" por similitud del vocablo que se emplea en las obras de túneles, cuando se finaliza la perforación: se dice que se ha hecho "el cale". (En los ensayos los fluidos calan el hormigón a través de los capilares).

La precisión del método habria podido ser aumentada por varias vias: permeando mayores 
cantidades de fluidos y/o empleando menores diferencias de presiones. Ello conllevaria mayor tiempo de dédicación a los ensayos (hasta el punto de sobrepasar la jornada laboral), y emplear instrumental de mayor precisión. El carácter pragmático que se ha pretendido dar al método, y el extenso plan de ensayos en curso (que supone el ensayo de varios miles de probetas), ha conducido a las condiciones experimentales indicadas.

Se han realizado repeticiones, en una misma probeta de la permeabilidad al agua, y al aire (en este caso humedeciéndolas tras el ensayo), obteniéndose datos similares, por lo que se dieron por buenas las condiciones experimentales.

Un mismo tipo de hormigón se ha ensayado haciendo las amasadas con varios días de intervalo, obteniéndose permeabilidades muy parecidas, por lo que la metódica se consideró repetitiva.

El método se experimenta haciendo amasadas de laboratorio para varios tipos de cemento; dosificado en distintas cantidades; con distintos tipos de áridos, conos y TM; varios tipos de cenizas volantes, en cantidades variables y molidas o no; empleo o no de varios aditivos plastificantes; adición o no de cantidades controladas de arcilla a la arena; variación de la proporción de arena respecto al total de áridos. (El producto de todas esta variables arroja los miles de probetas mencionado).

Al disponer de todos los datos, a todas las edades del ensayo (hasta 1 año), se procesarán los resultados permitiendo cuantificar las correlaciones enunciadas, asi como intentar establecer algunas nuevas con otras relaciones entre los componentes del hormigón que reflejen mejor, que las ya comentadas, el comportamiento de los hormigones con adiciones.

Circunstancias comunes en todos los ensayos han sido las siguientes:

— Los fluidos empleados en estos estudios han sido: agua y aire.

- La conservación de las probetas ha sido la normalizada.

Las limitaciones encontradas al método han sido las siguientes:

- Para tamaños de árido de $32 \mathrm{~mm}$ salen ya datos erráticos, por lo que cabe pensar que una primera limitación de tamaño sería la ya ensayada de $25 \mathrm{~mm}$. (Con probetas de dimensiones mayores que las empleadas no debieran presentarse estos datos, o con tapones menores, aunque aumentaría el tiempo de secado).

- Al cerrar el orificio con el tapón expansivo, se origina una tensión en la parte superior de la probeta, que puede llegar a romper por tracción, si el hormigón es de baja resistencia. Puede evitarse zunchando esa zona con las bridas adecuadas. Si el hormigón fresco exuda mucha agua, también puede fisurar por esa zona, al fraguar.

\section{GENERALIZACION DEL METODO "DEL CALE"}

Como ya se indicó al dar datos obtenidos por este método, había que hacer referencia a toda una serie de condiciones experimentales que se presuponian en el nombre del método. Si se pueden generalizar estos datos, de forma que no dependieran del tipo de probeta ni de las condiciones experimentales, podria dejarse de hacer referencia al método de ensayo, ya que se habrian obtenido parámetros inherentes al propio hormigón, dependiendo en teoria sólo del tipo de conservación a que se le hubiera sometido.

Un camino para la generalización se pormenoriza en las leyendas 1 a 8 . 


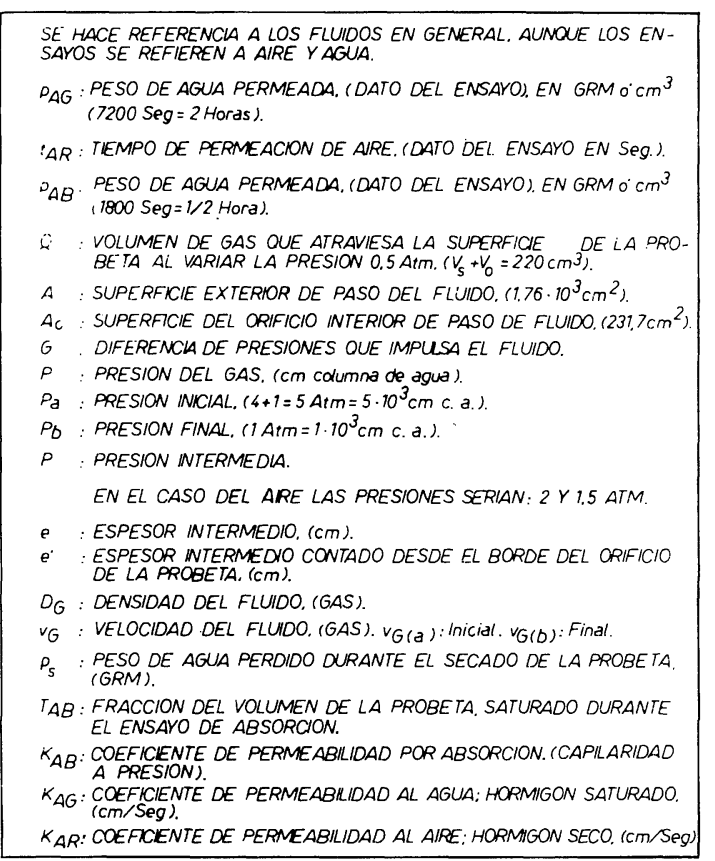

El significado fisico de $K$, es el caudal de fluido que atraviesa la unidad de superficie de una seccion de hormigón de espesor unidad, impulsado por una diferencia de presiones igual a la unidad (tiene las dimensiones de una velocidad). La correlacion de las cuatro ultimas variables con la resistencia, permiten la generalizacion del metodo, haciendo abstraccion del tipo de probeta utilizada, lo que permite comparacion con otros tipos de ensas. (La conservacion de probetas ha sido la normalizada, por ello los comparar los distinto tipos de dosificacion de hormigon y permiten pu

Leyenda 1
DATOS INTERMEDIOS NECESARIOS PARA EL CALCULO DE COEFICIENTES

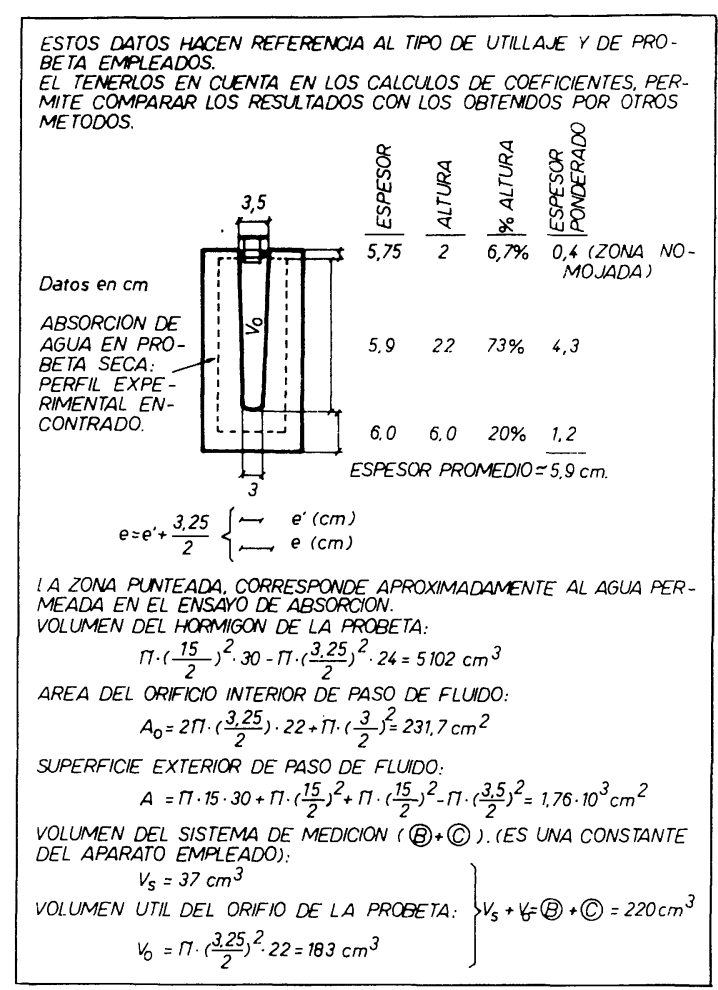

Leyenda 2

Estas deducciones se han segregado del texto de este escrito, para orillar más fácilmente su lectura por las personas no interesadas específicamente en el tema y, a la vez, para tratarla con suficiente detalle, de forma que pueda ser seguido paso a paso por las que sí les interese, en la confianza de que lo puedan contrastar con sus propios criterios y, en su caso, enriquecer o matizar lo expuesto, lo que de antemano agradecen los autores. No obstante conviene hacer las aclaraciones indicadas a continuación.

En las deducciones se recurre a la Ley de Gases Perfectos; a la Ecuación de Continuidad del transporte de fluidos y a la Ecuación de Darcy. Con ellas, y algunos datos experimentales, se da el mismo enfoque al cálculo de Coeficientes, tanto si el fluido es líquido como gas.

Esta última se aplica a la propagación de fluidos en medios porosos (por ejemplo: agua en los suelos). Puede enunciarse diciendo que el caudal del fluido es igual a la superficie de paso multiplicada por el gradiente de presiones que impulsa al fluido, multiplicando, a su vez, por una Constante llamada Coeficiente de Permeabilidad que depende de las caracteristicas del medio poroso. (En nuestro caso del tipo de hormigón).

A lo largo de la exposición se ve que juega un papel importante la forma de cómo se propaga el fluido en el interior de la probeta. Esto se ha podido comprobar sólo para líquidos, inyectando a las probetas agua coloreada y rompiéndolas al ensayo brasileño; se admite que los gases tendrán similar tipo de propagación, por lo que la resolución de la integral que queda afectada por este perfil se hace extensiva a cualquier fluido.

En el caso de gases, el planteamiento se complica al ser la presión inicial variable, en función del tiempo de ensayo. Se ha encontrado una fórmula empírica que satisface esos datos, y se ha 
PERMEABILIDAD A LOS FLUIDOS

- DATOS AUXILIARES

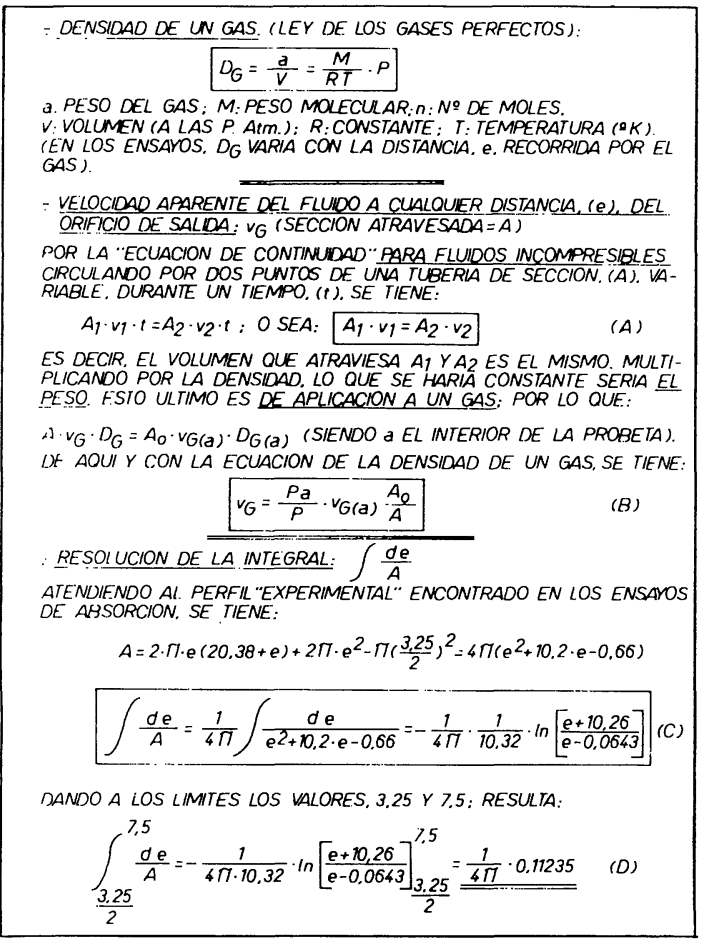

Leyenda 3
PERMEABILIDAD A LOS GASES (AIRE) DATOS AUXILIARES

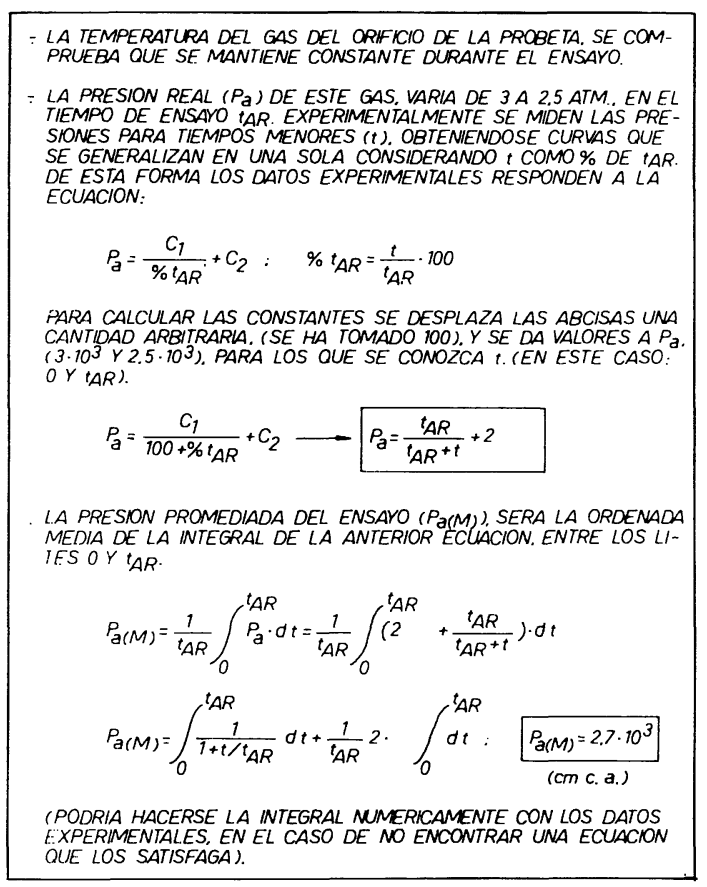

Leyenda 4

calculado la presión promedio. Se ha aceptado también que la temperatura del gas es constante, ya que la variación de la del gas contenido en el orificio, a lo largo del ensayo, es del orden de $1^{\circ} \mathrm{C}$, por lo que no influye en el planteamiento.

La absorción de liquidos (leyenda 6), se ha subtitulado "capilaridad a presión". En la leyenda 5, y tras el cálculo del coeficiente de permeabilidad al agua, se ha introducido un nuevo concepto $\left(T_{\mathrm{AB}}\right)$ definido en función del dato del ensayo de absorción de liquidos. (Parece interesante el contemplarlo, por lo que tiene de singular en los resultados experimentales, ya que no parece depender de la consistencia y del tamaño máximo, aunque sí de la resistencia y de los componentes del hormigón).

En la leyenda 9 se hace una estimación de los parámetros que parecen poder llevar a estimar un radio promedio de los capilares en el hormigón. Si ello es así, la singularidad apuntada para $T_{\mathrm{AB}}$ podría relacionarse con dicho radio, ya que para un mismo $T_{\mathrm{AB}}$ podria haber distintas permeabilidades, puesto que éstas están influidas por la superficie de paso, que incluiría este radio elevado al cuadrado. Todo ello parece inducir a pensar que, el tamaño de los capilares formados, puede estar influido por los materiales empleados, esencialmente el radio capilar a igualdad de longitud de los que componen el hormigón, siendo menores cuanto menores sean los coeficientes de permeabilidad - relación ésta sugestiva - por las implicaciones de todo tipo que tiene para prever las prestaciones del hormigón, en función de sus componentes. Datos recientes apuntan que $T_{\mathrm{AB}}$ depende sólo de conglomerante (cemento + adición), independiente incluso del tipo de arena ensayada.

En la leyenda 9 se hace también un esbozo de enfoque de la penetración de liquidos en el hormigón seco, por capilaridad, al objeto de intentar sugerir nuevas aplicaciones con ensayos basados en los principios expuestos.

De cualquier forma conviene recalcar que una cosa son los datos de los ensayos y otra la 


\section{COEFICIENTE DE PERMEABILIDAD}

A LOS LIQUIDOS (AGUA)

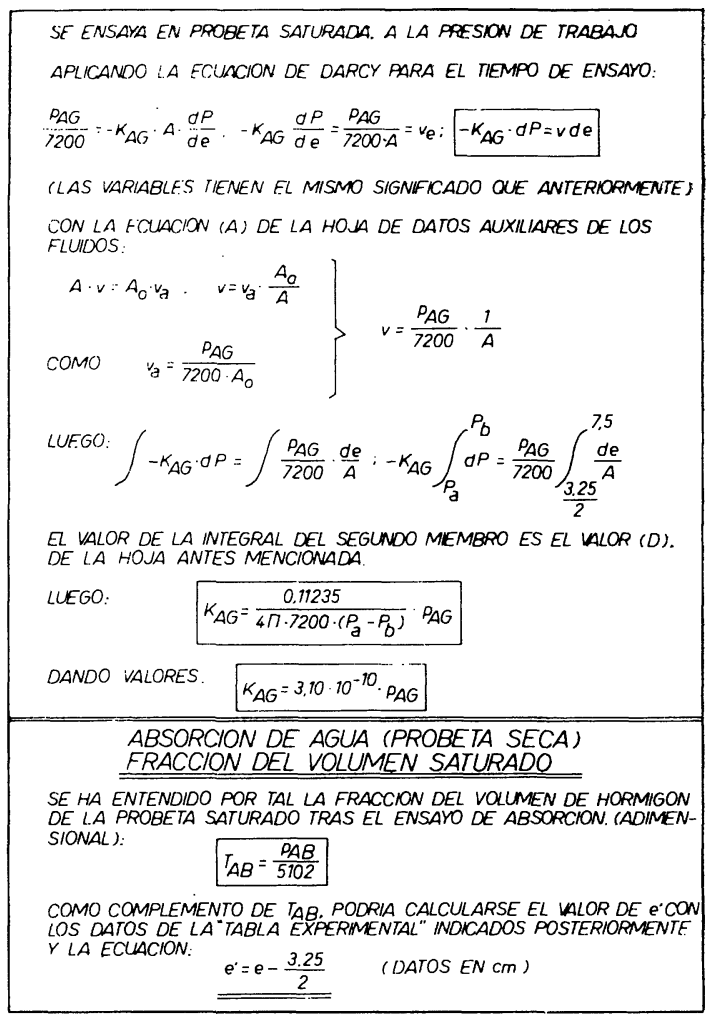

Leyenda 5
PERMEABILIDAD POR ABSORCION

CAPILARIDAD A PRESION (AGUA)

SE ENSAYA EN PROBETA SECADA. EN LAS CONDICTONES DEL METOOO. SE CALCULA ADMMTIENDO QUE LA PENETRACON DE LOUDO SIGLE UNA RA EL AGLA. ESTE PERFI ORIGINA UN VOLUMEN DE HORMIGON SAFURADO DE AGUA QUE LLAMAREMOS VPE.
EL ESPESOR DE HORMHGON DEL "PERFLL EXPERIMENTAL": SE DA EN FUNCION DEL DATO DE ESTE ENSAYO. (PAB). Y DE LA PERDIDA DE PESO EN EL SECADO IPS. SE DAN VALORES TABULDOS. CON $L O$ OUE PUEDE INTERPELACSE DATOS INTERMEDDOS, POR SER LABORIOSO FL TE PROPORCIONALOAD:

$$
\begin{array}{|l|c|c|c|c|c|c|c|}
\hline P_{A B} & V_{P E} \\
\hline P_{S} & \frac{P_{A B} / P_{S}}{5102} & 0 & 0.05 & 0.10 & 0.15 & 0.25 & 0.40 \\
\hline \mathrm{e}(\mathrm{cm}) & 1.63 & 2.47 & 3.07 & 3.55 & 4.33 & 5.25 \\
\hline
\end{array}
$$

APLICANDO LA ECUACION DE DARCY. PARA EL TIEMPO DE ENSAYO

$\frac{p_{A B}}{1800}=-K_{A B} \cdot A \cdot \frac{d P}{d \mathrm{e}}:-K_{A B} \frac{d P}{d \mathrm{e}}=\frac{P_{A B}}{1800 \cdot A}=v:-K_{A B} \cdot d P=v d \mathrm{e}$

(LAS VARIABLES TIENEN EL MISMO SIGNIFICADO OUE ANTERIORMENTE) CON LA ECUACION (A) DE LA HOLA DE LOS DATOS AUXILIARES DE LOS

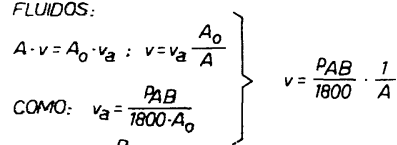

LUEGO: $\int_{P_{a}}^{P}-K_{A B} \cdot d P=\int_{\frac{3.25}{2}}^{e} \frac{P_{A B}}{1800} \cdot \frac{d e}{A} ;-K_{A B} \int_{P_{a}}^{P_{D}} d P=\frac{P_{A B}}{1800} \int_{\frac{3.25}{2}}^{e} \frac{d e}{A}$

SE ADMITE QUE $P=P_{b}=1$ ATIm. (PRESION ATMOSFERICA)

EL VALOR DE LA NTEGRAL DEL SEG UNDO MIEMBRO. ES EL VAIOR (D). DE LA HONA ANTES MENCIONADA.

LUEGO: $K_{A B}=\frac{-P_{A B}}{1800\left(P_{a}-P_{b}\right)} \cdot \frac{1}{4 \pi \cdot 10.32} \cdot \ln \left[\frac{e+10.26}{e-0.0643}\right]_{\frac{3.25}{2}}^{e}$

DANDO VALORES

$$
K_{A B}=-1.07 \cdot 10^{-9} \cdot\left(\ln \frac{e+10.26}{e-0.0643}-2.030\right) \cdot p_{A B}
$$

EL VALOR DE I SE NTERPOLA EN LA TABLA.

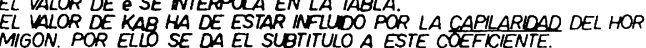

Leyenda 6

PERMEABILIDAD A LOS GASES (AIRE)

SE ENSAYA EN PROBETA SECADA. EN LAS CONDICIONES DEL METODO BLE:

$$
\frac{Q}{t_{\Delta R}}=-K_{A R} \cdot A \cdot \frac{d P}{d \mathrm{e}} ; \quad-K_{A R} \cdot \frac{d P}{d \mathrm{e}}=\frac{Q}{t_{A R} \cdot A}=v_{G}
$$

EL SIGNO MENOS SE DEBE A QUE EL GRADIENTE DISMINUVE EN LA DIRECCION DE PASO DE GAS. SIENDO Q: A Y VG: EL VOLLMEN. (MEDIDO A LA CORRESPONDIENTE PRESION), LA SUPERFICIE DE PASO, Y LA VE SALIDA LLAVISOSIDAD NO INFLUYE AL NO DEPENDER DE LA PRE SION Y ADMITIRSE TEMPERATURA CONSTANTE, YA OUE SU VARIACION ES DEL ORDEN DE $1^{\circ} \mathrm{C}$

LA ECUACION (A) DE LA HONA ANTERIOR DE DATOS AUXILIARES, PUEDE APLICARSE AL PUNTO DE SALIDA DEL ORIFICIO (a). SE CALCULA $\checkmark G(a)$ CONSIDERANDO COMO PRESION LA PROMEDIADA: Pa(M).

$$
v_{G}=\frac{P_{a}(M)}{P} \cdot v_{G(a)} \cdot \frac{A_{0}}{A}
$$

APLICANDO (B') EN (1), SE TIENE:

$$
\begin{aligned}
& -K_{A R} \cdot P \cdot \frac{d P}{d \mathrm{e}}=P_{a(M)} \cdot v_{G(a)} \cdot \frac{A_{0}}{A} \\
& -K_{A R} \int P d P=P_{a(M)} \cdot v_{G(a)} \cdot A_{0} \cdot \int \frac{d e}{A}
\end{aligned}
$$

LA ECUACION (2) PUEDE INTEGRARSE PARA LIMITES CONOCIDOS. PROMEDIO DEL ORIFICIO Y LA PRESION EXTERIOR (P(D) OUE VALE 1000.

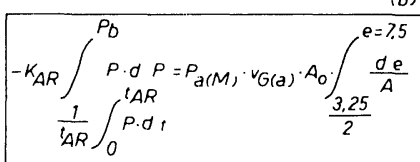

EN LA HOJA ANTERIOR DE DATOS AUXILIARES SE RESOLVIO LA INTE GRAL $\int \frac{d e}{A}$ OUE ENTRE LOS LIMITES INDICADOS DA UN VALOR (D).

SIGUE..

Leyenda 7

\section{PERMEABILIDAD A LOS GASES (AIRE)} EL LIMITE INFERIOR DE LA PRIMERA INTEGRAL DE (3). SE ACEPTA QUE
EQUINALE A Pa(M), CUYO VALOR, YA CALCULADO. ES DE $27.10^{3}$ PARA CALCULAR VG(a). SE CALCULA EL VOLUMEN TOTAL DEL GAS, (Q). EN EL SISTEMA DE MEDICION, (B) + (D), DONDE SU PERDIDA ORIGINA UN DESCENSO DE PRESION DE $(2-1,5)=0,5$ ATM, Y PARA CONDICIONES NORMALES, $\left(20^{\circ} \mathrm{C}\right.$ Y $\left.760 \mathrm{~mm} \mathrm{Hg}=P(D)\right)$, SE TIENE:
NOR

$Q_{1}=\frac{0.5 \cdot \text { VOLUMEN }(B)+(C)}{R \cdot(273+20)} \cdot($ VOLUMEN MOLAR $)$

$Q_{1}=\frac{0,5 \cdot 220}{0.082 \cdot(273+20)} \cdot 24,0=110 \mathrm{~cm}^{3}$ (EN ESTE APARATO)

ADMITIENDO COMO PRESION PROMEDIO EN EL INTERIOR DE LA PROBE TA LA MEDIA ENTRE Pa Y PD TANTO AL PRINCIPIO COMO AL FINAL DEL ENSAYO. EL DESCENSO DE PRESION ES DE 1/4 ATM. SI EL VOLU-
MEN OCUPADO POR EL GAS ES PS EL VOLUMEN DEL GAS OUE SALE ES: $P_{S} / 4$. CON ELLO QUEDA CONOCIDO EL VALOR:

$$
v_{G(a)}=\frac{Q_{1}+p_{S} / 4}{{ }^{t} A R \cdot A_{0}}
$$

INTEGRANDO LA (3) SE TIENE

$$
\kappa_{A R} \frac{P_{a(M)^{2}}-P_{D}^{2}}{2}=P_{a(M)} \cdot \frac{Q+P_{S} / 4}{A_{0} \cdot t_{A R}} \cdot A_{0} \cdot \frac{1}{4 \pi} \cdot 0,11235
$$

IUEO.

$$
K_{A R}=\frac{\left(Q+P_{S} / 4\right) \cdot P_{a}(M) \cdot 0,11235}{2 \pi \cdot\left(P_{a}(M)^{2}-P_{b}^{2}\right)} \cdot \frac{1}{t_{A R}}
$$

DANDO VALORES EN (6):

$$
K_{A R}=\frac{7.68 \cdot 10^{-6}\left(110+\rho_{S} / 4\right)}{{ }^{t} A R}
$$

Leyenda 8 
OTRAS VARIABLES A ESTUDIAR (POSIBLES ENFOQUES)

PRESION POR CAPILARIDAD (PC), Y RADIO PROMEDIO DE LOS CAPILARES ( $r$ )

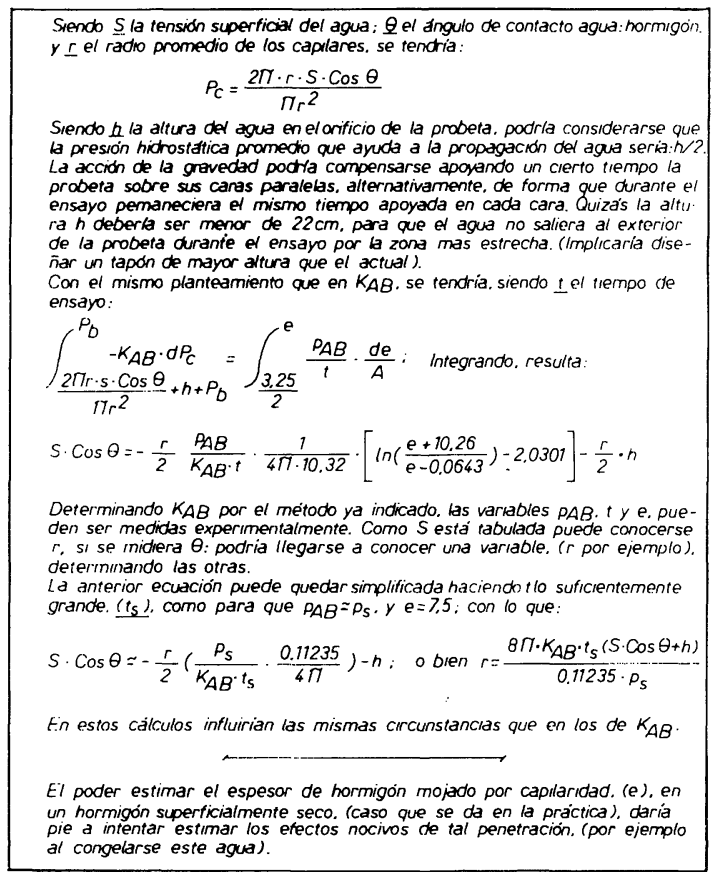

Leyenda 9
ECUACIONES PARA EL CALCULO DE LOS COEFICIENTES

\begin{tabular}{|c|c|c|}
\hline $\begin{array}{l}\text { SE RESUMEN LAS ECUACION } \\
\text { FICADO DE LAS VARIABLES } \\
\text { DE LAS FORMULAS EMPLEA }\end{array}$ & $\begin{array}{l}\text { WTRADAS } \\
\text { ERVIENEN }\end{array}$ & $\begin{array}{l}\text { ECUERDA EL SIGNI- } \\
\text { LAN ACLARACIONES }\end{array}$ \\
\hline$P_{A G}:$ PESO DE AGLA PERM & $G R M:\left(\mathrm{cm}^{3}\right)$. & \\
\hline${ }^{\prime} \triangle R:$ TIEMPO DE PERMEACIO & AIRE (SEG). & \\
\hline$\rho_{A B}:$ PESO DE AGUA PERME & $\angle A, A B S O F$ & $N\left(G R M ; \mathrm{cm}^{3}\right)$ \\
\hline $\mathrm{e}:$ : ESPESOR DE HORMGO & MEADO EN & BSORCION $(\mathrm{cm})$. \\
\hline$D_{S}:$ PESO PERDIDO EN EL & O (GRM : Cn & \\
\hline$K_{A G .} \begin{array}{l}\text { COEFICIENTE DE PERM } \\
(\mathrm{cm} / \mathrm{Seg}) .\end{array}$ & $D A D A L A G L$ & RMIGON SATURADO \\
\hline$K_{A R}:$ COEFCIENTE DE PERM & $A D$ AL AIRE & MIGON SECO $(\mathrm{cm} / \mathrm{Seg})$ \\
\hline$K_{A B}$. PERMEABILDAD POR A & ON: CAPRAR & A PRESION (cm/Seg) \\
\hline$T_{A B}: A B S O R C I O N D E$ AGUA. & ON SECO. & SDIMENSIONAI) \\
\hline$K_{A G}=3.10 \cdot 10^{-10} \cdot P_{A G}$ & $K_{A R}=\frac{7,6}{2}$ & $\left(P_{S} / 4+170\right)$ \\
\hline$K_{A B}=-1,07 \cdot 10^{-9} \cdot\left(\ln \frac{e}{e-}\right.$ & $2.030) \cdot P_{A B}$ & $T_{A B}=\frac{P_{A B}}{5102}$ \\
\hline $\begin{array}{l}\text { - LAS CONSTANTES DE LAS } \\
\text { LLAJE Y METODO DE ENSA } \\
\text { OUE NO SE MIDEN EN SITL } \\
\text { SO DE KAR ES INERMEDI } \\
\text { ADEMAS INFLUYEN LAS CD }\end{array}$ & $\begin{array}{l}\text { IONES HACE } \\
\text { PLEADO. EN } \\
\text { DE REGIME } \\
\text { DUE SE PRC } \\
\text { ITES DEL A }\end{array}$ & $\begin{array}{l}\text { EFERENCA AL UTI- } \\
\text { ECAL TAB Y KAB, YA } \\
\text { STACIONARIO. EL CA- } \\
\text { IIA LA PRESION; } \\
\text { ITO. }\end{array}$ \\
\hline $\begin{array}{l}\text { - I.OS COEFICIENTES OBTENID } \\
\text { PLEADOS: EL DATO DE UN } \\
\text { PONENTE. }\end{array}$ & $\begin{array}{l}\text { ENDEN DE } \\
\text { VARIA SI }\end{array}$ & $\begin{array}{l}\text { MATERLALES EM- } \\
\text { BA ALGUN COM- }\end{array}$ \\
\hline$=$ EN CLALOUIER CASO INFLUI & CONSERVAC & DE LAS PROBETAS. \\
\hline $\begin{aligned} \text { - } & \text { EL DATO KAG PARECE E } \\
& \text { NE REFERRIRLOS AL ME } \\
& \text { HAYA EXPERIENCIAS DE }\end{aligned}$ & $\begin{array}{l}\text { VERALIZA } \\
\text { EADO } \\
\text { ION CON }\end{array}$ & $\begin{array}{l}\text { S DEMAS CONVIE- } \\
\text { ALE) MIENTRAS NO } \\
S \text { METODOS. }\end{array}$ \\
\hline $\begin{array}{l}=\text { EN CLLLQUIER CASO LO } \\
\text { SI MISMOS DISTINTOS } T\end{array}$ & $\begin{array}{l}\text { LOSE } \\
\text { RMIGON }\end{array}$ & COMPARAN POR \\
\hline
\end{tabular}

Leyenda 10

generalización de éstos. En cuanto a los datos, su validez para comparar distintos tipos de hormigón es evidente. $\mathrm{Y}$ en cuanto a la generalización de los coeficientes obtenidos en la leyenda 10 (que es un resumen de las fórmulas a aplicar) se indica la conveniencia de comparar con otros - encontrados por otros métodos-, para comprobar si realmente sólo depende del tipo de conservación a que se haya sometido al hormigón. (A este respecto, podría pensarse hacer ensayos de información extrayendo testigos de $15 \mathrm{~cm}$ de diámetro, perforándolos con orificio de $3,5 \mathrm{~cm}$ de diámetro, que se taponaria con dos tapones expansivos, con lo que se conformaría una probeta sobre la que efectuar ensayos de permeabilidad).

\section{ALGUNOS DATOS EXPERIMENTALES}

En los gráficos del 1 al 4 se indican los resultados experimentales obtenidos para los hormigones descritos en el encabezamiento. En los n.os 2, 3 y 4, las resistencias se han acotado a 125 y $375 \mathrm{~kg} / \mathrm{cm}^{2}$, y se ha puesto una escala auxiliar en MPa. Se han representado los cuatro datos que se obtienen con las fórmulas de la leyenda 10.

La curva de áridos se mantuvo en todos los ensayos, y era de tipo continuo.

A titulo comparativo, y empleando en las abscisas una escala totalmente arbitraria, se indican en el gráfico 1 , los resultados obtenidos para los hormigones con $250 \mathrm{~kg} / \mathrm{m}^{3}$ de un cemento PA-350, con consistencias plástica y fluida, y tamaños máximos de áridos 25 y $12 \mathrm{~mm}$ (este último suele ser el menor de los tamaños máximos empleados en la elaboración de hormigón). Los datos correspondientes a este último hormigón pueden servir de pauta para contrastar las permeabilidades, con la correspondiente al hormigón referenciado en la normativa, como adecuado para ciertos elementos de obras. En los gráficos 2,3 y 4 se evidencia que cualquiera de los hormigones contemplados no llegan al umbral deducido anteriormente. 
PERMEABILIDAD DEL HORMIGON

DE DOSIFICACION

$250 \mathrm{~kg} / \mathrm{m}^{3}$ DE PA-350

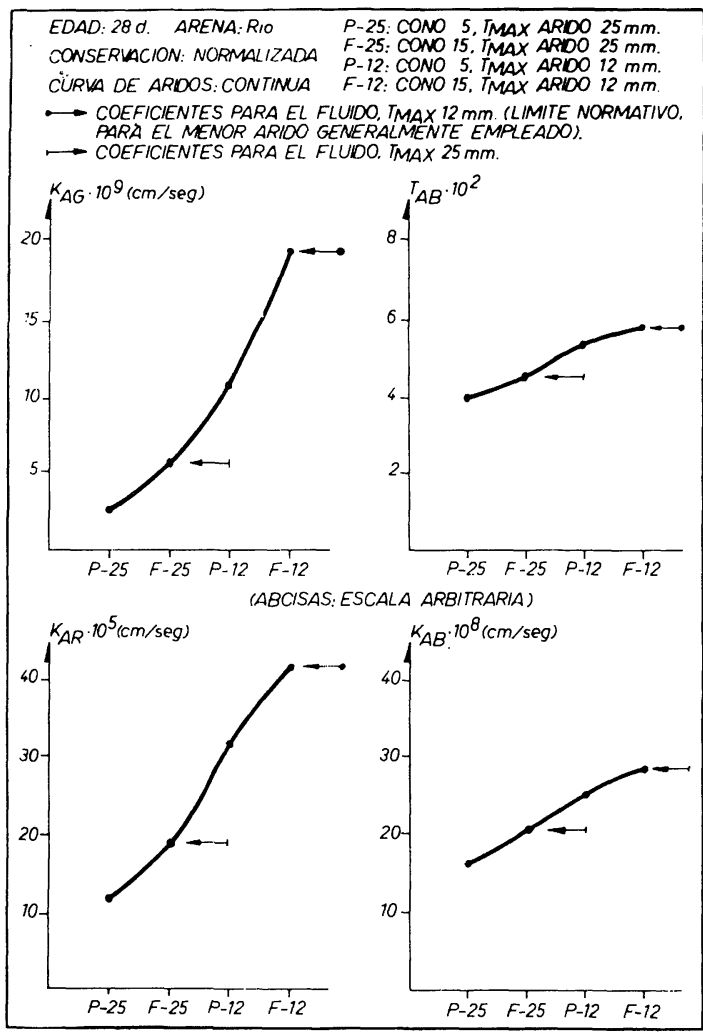

Gráfico 1
PERMEABILIDAD FRENTE A RESISTENCIA A COMPRESION

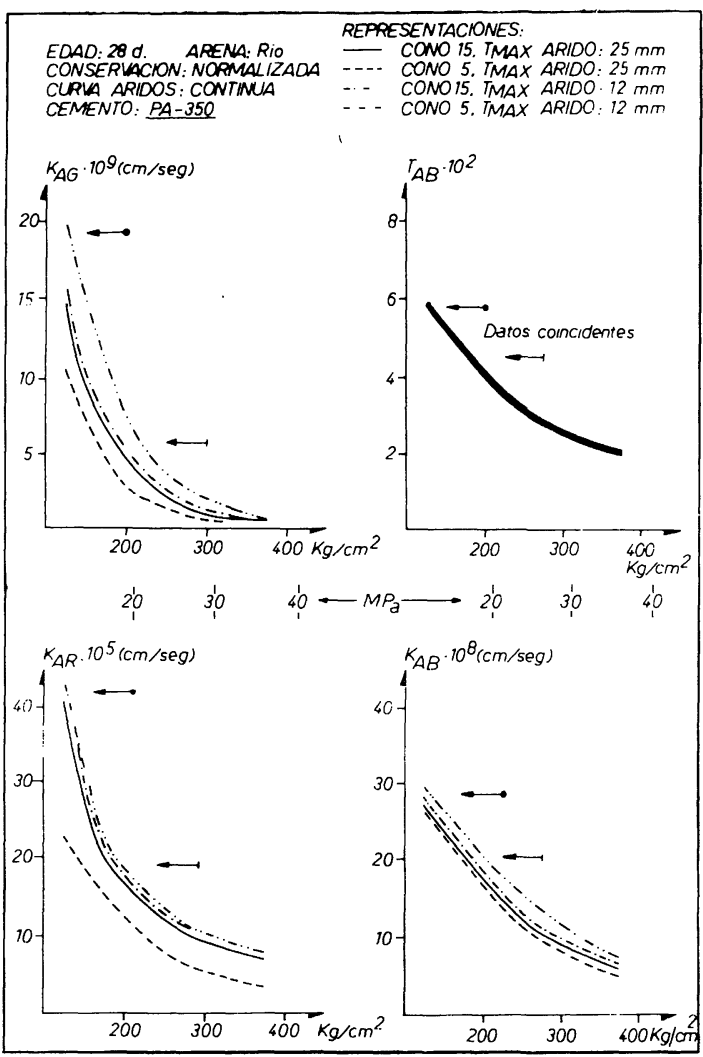

Gráfico 2

Es remarcable la concordancia entre los gráficos 1 y 2 en cuanto a que la influencia en la permeabilidad de la variación en el tamaño máximo del árido y de la consistencia se mantiene, para el margen de resistencias considerado, con la excepción de la singularidad de $T_{\mathrm{AB}}$, ya comentada.

En el gráfico 3 se detecta una curiosa circunstancia: es la distinta permeabilidad de un P-450 y un PA-350 elaborados con el mismo clínker con la diferencia de la incorporación en fábrica de una adición. Las permeabilidades, para hormigones de la misma resistencia, son mayores para el cemento con adición.

Con el mismo P-450, pero adicionando directamente en la hormigonera otra adición (cenizas de Cercs), se obtuvieron los datos del gráfico 4, que se comparan con los .correspondientes al PA-350 del gráfico 3, encontrándose aún más diferencia de permeabilidades en el gráfico 4 que en el 3 .

Estas tendencias en las variaciones de la permeabilidad se confirman con muchos otros datos de hormigones, con otros tipos de áridos, con $\mathrm{y} \sin$ varios tipos de plastificantes habituales en la industria del hormigón para otros tamaños máximos y otras consistencias. No mejora, sino al contrario, el efecto relativo de las adiciones contempladas en los gráficos 3 y 4 al molerlas previamente.

Otro parámetro que afecta a la permeabilidad es la curva granulométrica de conjunto de áridos. Se ha ensayado variar el porcentaje de las arenas ensayadas, respecto al total de áridos, encontrándose un óptimo que corresponde al empleado en los ensayos. Porcentajes menores hacen aumentar la permeabilidad, y porcentajes mayores también, y en mayor cuantia; todo ello 
PERMEABILIDAD FRENTE A RESISTENCIA A COMPRESION

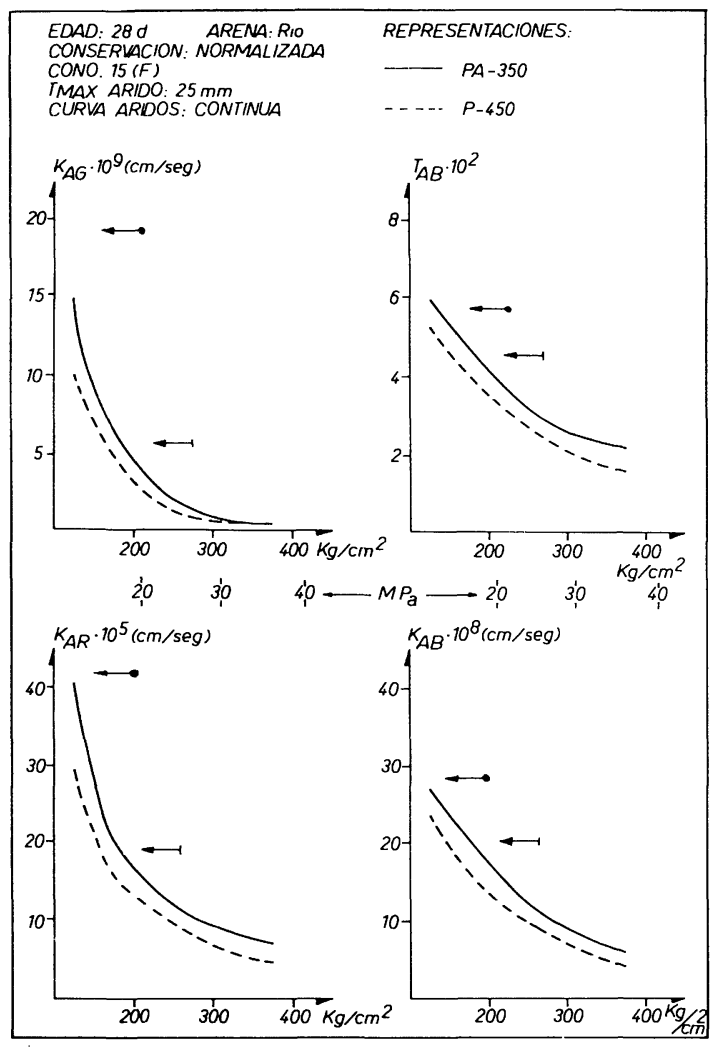

Gráfico 3
PERMEABILIDAD FRENTE A RESISTENCIA

A COMPRESION

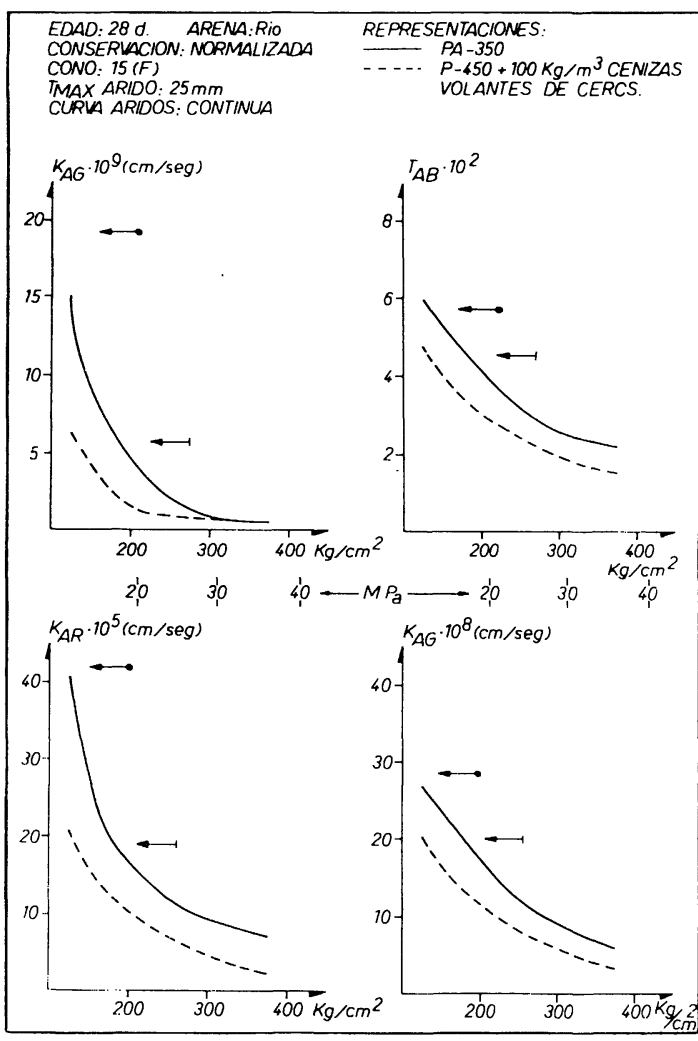

Gráfico 4

para la potencia de compactación empleada al elaborar las probetas. (Picado con barra, según norma).

Otra circunstancia que se da en la práctica es la presencia de arcilla en los áridos. Para comprobar si influía en la permeabilidad se añadieron proporciones conocidas de arcilla a la arena empleada, observándose que disminuye apreciablemente la resistencia pero que, partiendo de este último dato, la permeabilidad obtenida era la previsible por las correlaciones provisionales de que se dispone.

El comportamiento de los aditivos plastificantes en la inpermeabilidad es muy positivo, en especial en hormigones con las adiciones ensayadas.

\section{CRITERIOS PARA CUANTIFICAR LAS PRESTACIONES DEL HORMIGON}

En la actualidad, el hormigón se especifica por su Resistencia, Consistencia y Tamaño Máximo de Arido. Cuando se quiere acotar alguna otra característica que no puede medirse directamente (impermeabilidad, durabilidad...) suelen ampliarse estas especificaciones a otras tales como: el contenido de cemento, o la relación entre la cantidad de algún compuesto quimico presente en el hormigón y alguno de sus componentes, generalmente el cemento.

De lo indicado hasta ahora se deduce que las tres especificaciones actuales afectan a casi todas las propiedades del hormigón. Junto con la impermeabilidad (que tienen en cuenta sus distintos componentes) se le adicionan, prácticamente, todas las que pueden alterarlas por causas exógenas al hormigón. 
La más clásica de las relaciones entre componentes es quizás la relación agua-cemento. Pues bien, en los hormigones que llevan adición, tanto si ésta se incorpora en la fábrica de cemento, como en la planta de hormigón, deja de tener validez, ya que no refleja el comportamiento de dichos hormigones.

Se indican las relaciones agua-cemento de los hormigones dados, como ejemplo de estas experiencias, donde se ve la incoherencia entre dicha relación y la resistencia: para una misma resistencia, las relaciones entre el agua y el cemento de cada amasada de prueba debería ser la misma. (Se dan las relaciones respecto a la del hormigón tomado anteriormente como patrón de comparación, el D-250-F-12, para hacer patente el error proporcionalmente a ella).

Los datos se dan referidos al hormigón de $200 \mathrm{~kg} / \mathrm{cm}^{2}$ de resistencia a 28 días.

\begin{tabular}{|c|c|c|c|c|c|}
\hline \multicolumn{4}{|c|}{ PA-350 } & \multicolumn{2}{|r|}{$\mathrm{P}-450$} \\
\hline $\mathrm{F}-12$ & $\mathrm{P}-12$ & F-25 & P-25 & F-25 & F-25 (Con Cenizas) \\
\hline 1 & 1,00 & 1,09 & 1,13 & 1,35 & 1,72 \\
\hline
\end{tabular}

Las diferencias debidas a la consistencia y al tamaño máximo del árido, con PA-350, no son definitorias, pero sí empiezan a serlo cuando se pasa a P-450 y más aún con el empleo de adición.

El caso es peor cuando pretende resumirse el comportamiento del hormigón con la relación de algún componente quimico y algún componente del hormigón, en general el cemento. En el caso de los hormigones con adición suele cometerse el error siguiente: si la adición se añade en la fábrica de cemento, se cuenta como cemento, con lo que el denominador de la fracción tiene un cierto valor. Si la adición se añade en la planta de hormigón, no se cuenta como cemento, con lo que dicho denominador tiene otro valor. (En ambos casos la composición química del hormigón es idéntica, a igualdad de las adiciones). Al ser las premisas falsas, son falsas las conclusiones que de ellas se deduzcan, haciendo preveer prestaciones distintas para hormigones similares, lo que evidencia que se plantea un sofisma.

Para dar nueva luz al tema podria recordarse, como causa primigenia generalmente aceptada, el parámetro que parece incidir más en las propiedades del hormigón, y que es el agua sobrante tras la hidratación.

Sabido es que el hormigón debe amasarse con mucha más agua de la estrictamente necesaria para hidratarse, permitiendo su puesta en obra. El agua sobrante acaba evaporándose y creando la red de capilares que hace permeable al hormigón. La singularidad señalada, respecto a los datos de $T_{\mathrm{AB}}$, indican la buena correlación que parece haber entre el volumen de dicha red capilar y la resistencia del hormigón.

Se están recopilando datos experimentales los cuales parecen apuntar que, por este camino, podrian encontrarse relaciones que satisfacieran mejor las características del hormigón que las que actualmente suelen emplearse.

Es interesante recordar que no hace tanto tiempo el hormigón se referenciaba por relaciones volumétricas entre sus componentes. Era clásico el $1: 2: 3$, actualmente no; por ello cabe pensar en otros cambios que actualicen las relaciones entre sus componentes.

\section{CONCLUSIONES}

El método expuesto ha permitido cuantificar las permeabilidades de numerosos tipos de hormigón, y aportar nuevas experiencias que abundan en el sentido de inducir a reconsiderar los 
criterios clásicos de pretender enriquecer las especificaciones técnicas del hormigón en base a fijar minimos a alguno de sus componentes, o a acotar las relaciones entre ellos. El criterio podria ser el de fijar las permeabilidades al hormigón (o fijarlas mediante la oportuna correlación con la resistencia a compresión). Estas limitaciones podrian ser fijadas en función del medio en el que el hormigón deba desarrollar su acción resistente.

Con estos planteamientos podria proyectarse, controlarse y/o construirse, diseñando el hormigón de forma más acorde con las prestaciones que de él se esperen.

Se agradece a los Sres. Fco. Javier Martínez de Eulate (de ANEFHOP), y Eduardo Herrero Nuñez (del CIDA), la atenta lectura de originales, y los comentarios al método expuesto.

\section{publicaciones del i.e.t.c.c.}

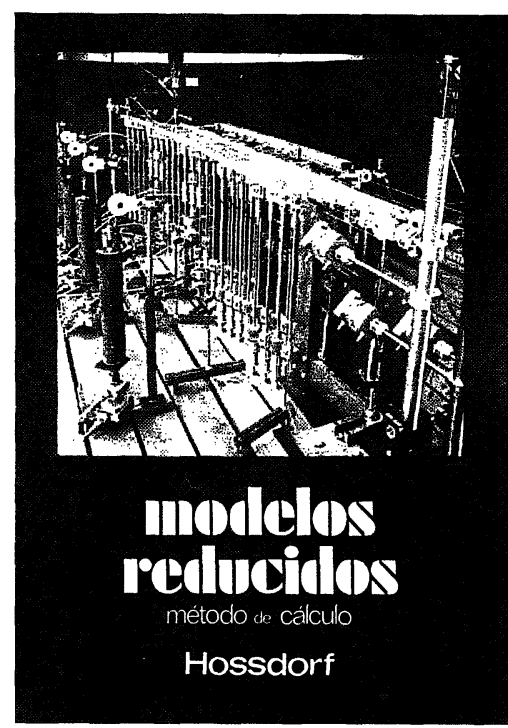

Modelos reducidos. Método de cálculo

H. Hossdorf, Ingeniero Civil

La técnica de los ensayos en modelos reducidos de estructuras sufre hoy dia una decisiva metamorfosis
Hasta hace poco era un medio más bien de artesania, que no siempre era tomado en serio por los académicos teorizantes oara comprender el comportamiento resistente de las estructuras complejas y al que se acudió las más de las veces, como a un último remedio debido a sus indiscutibles insuficiencias. Sin embargo, en poco tiempo y gracias a su conexión con los ordenadores digitales, se ha transformado en un instrumento cientificamente valioso, que no puede quedar a un lado en la práctica diaria del Ingeniero Proyectista.

Un volumen encuadernado en cartoné plastificado con lomo de tela, de $17 \times 24 \mathrm{~cm}$, compuesto do 250 páginas, 158 figuras $y$ fotografias.

Precios: 1.800 ptas.; $\$$ USA 26.00

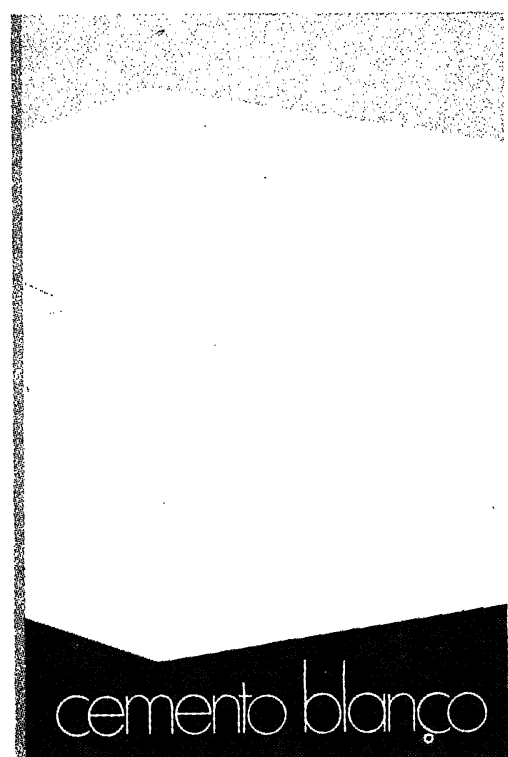

\section{Cemento blanco}

Julián Rezola

Ingzeniero Quimico Dipl. I. Q. S.

Sabido es que existe una extensa y documentada bibliografia sobre el cemento gris: en cambio, no puede decirse lo mismo acerca del cemento portland blanco, ya que los escritos existentes se refieren tan sólo a algunas peculiaridades que le distinguen de aquél.

El autor nos ofrece sus profundos conocimientos y su larga experiencia tanto en laboratorio como

La parte descriptiva del libro se complementa con gráficos, diagramas y fotografias de gran utilidad, destinados a conseguir la aplicación apropiada de este aglomerante.

Un volumen encuadernado en cartoné policerado, de $17,4 \times 24,3 \mathrm{~cm}$, compuesto de 395 páginas

Precios: España, 1.700 ptas.: extranjero, $\$ 34$

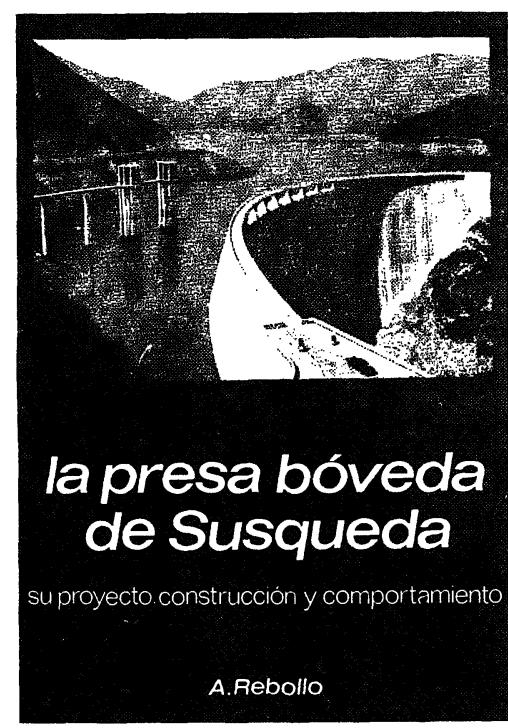

La presa bóveda de Susqueda

A. Rebollo,

Dr. Ingeniero de Caminos

El esfuerzo del constructor de presas se sitúa por su pretension de perennidad, a contracorriente de las tendencias de la civilización actual, caracterizada por lo fungible. Pueden evocarse las 10.000 grandes. presas en funcionamiento o en construcción que estan envejeciendo y reclaman los cuidados gerontologicos para mantener y perfeccionar su envicio y garanizar su inalienable pretensión de perennidad. En la medida en que todas nuevas riesgos rón aumentan con envejeciniento las presas es todo un emplazo. de Arturo Rebollo en este terreno marca un camino a seguir para todos los que aman su propia obra con la devoción paternal que el ha puesto en Susqueda.

Un volumen encuadernado en cartone plastificado con lomo de tela, de 18 , $24,5 \mathrm{~cm}$ compuesto de 408 páginas, 330 figuras $y$ fotografias $y 39$ tablas. Precios: 1.700 ptas.; extranjero, \$ USA 24.00 